\title{
A Practical FPGA-Based LUT-Predistortion Technology For Switch-Mode Power Amplifier Linearization
}

\author{
Technological Platforms Section, Department of Electronic systems \\ Aalborg University, DK-9220, Denmark \\ U. Cerasani, Y. Le Moullec, Tian Tong \\ Email\{ylm@es.aau.dk, tt@es.aau.dk \}
}

\begin{abstract}
In the context of wireless communications, power amplifiers raise a number of issues. Mastering the linearity vs. efficiency trade-off is not trivial. Switch-mode power amplifiers can provide high power efficiency. However, they exhibit significant nonlinearities, which make them difficult to apply in modern high linear modulation wireless systems. In order to enable their use in such systems, a LUT-based predistortion technique targeting switch-mode PA linearization is introduced. Particularly the predistortion function derivation for handling the whole input interval of the nonlinearity predistortion is described. A $0.18 \mu \mathrm{m}$ CMOS Class $\mathrm{F}$ power amplifier is used as the study-case for testing and evaluating the proposed approach. Matlab and DSPBuilder blocks targeting a Stratix II FPGA simulation results show that the proposed LUT-based predistortion technique effectively improves the linearity of the switch-mode PA with an average EVM of less than $2 \%$.
\end{abstract}

Index Terms-Switch-mode power amplifier, digital linearization, LUT, wireless communications

\section{INTRODUCTION}

In a wireless transmitter chain, the Power Amplifier (PA) is the block that gives the modulated signal the necessary power for propagation once it leaves the antenna. For higher power applications, such as GSM and UMTS, it is important that the PA utilizes most of the DC power it drains from the DC power supply source in the process of amplification. This is highly desirable, for both handheld devices and base stations devices. With the current public debate on global warming, saving as much power as possible is important for any application - also in the wireless communication industry. There is always a tradeoff between linearity and efficiency among different classes of PAs, and this is one of main design challenges to overcome.

The high Power Added Efficiency (PAE) of switch-mode PAs is very attractive for mobile and portable systems. However, switch-mode PAs have always raised concerns regarding their practical applications in modern communication systems because of their significant nonlinearity. To efficiently use frequency resources and to achieve high data-rates, modern wireless communication systems almost always use high order modulations schemes, including both phase and envelope modulation. With the significant nonlinearity and the complex Amplitude-toAmplitude (AM-AM) and Amplitude-to-Phase (AM-PM) features, switch-mode PAs can not easily be linearized with traditional linearization technique. A high order polynomial based predistortion technique is frequently mentioned in various publications as e.g. in [1-2-3]. This technique involves large amount of analog blocks such as multiplier, Variable Gain Amplifier (VGA) and analog adder. The performance stability and accuracy of the analog blocks impact the accuracy of the predistortion severely, which leads to a serious predistortion performance degradation.

A number of digital predistortion techniques have been published, as e.g. in [4-8], but most of them focus on linear power amplifiers, therefore they are either not so efficient or only focus on a narrow interval. Switch-mode PAs feature a significant nonlinearity on the whole input interval, thus those techniques can not be used for switch-mode PAs. New methods and techniques have to be devised to improve the current linear PA oriented technique.

This paper presents a LUT-based technique for switchmode PA linearization. A polar configured Class $\mathrm{F}$ switchmode PA is used as the study-case to test and validate the proposed technique. A specifically designed predistortion function is derived and implemented. Altera's DSP-Builder library for Simulink is used to implement the proposed linearization approach on a Stratix II FPGA. The simulation setup is based on Matlab and Simulink. The simulation results, in particular the spectrum and EVM analyses, show that a Class F PA can be effectively linearized by the proposed approach.

\section{THEORY}

In this work, the design and implementation of a FPGAbased digital predistorter is investigated for switch-mode PAs. The proposed system-level architecture, shown in Fig. 1, consists of 4 blocks: a lookup table that contains pre-distortion coefficients, an address decoding block for the coefficient selection, the pre-distorter and a multiplier. The purpose of the multiplier is to apply the predistortion to the input signal. 


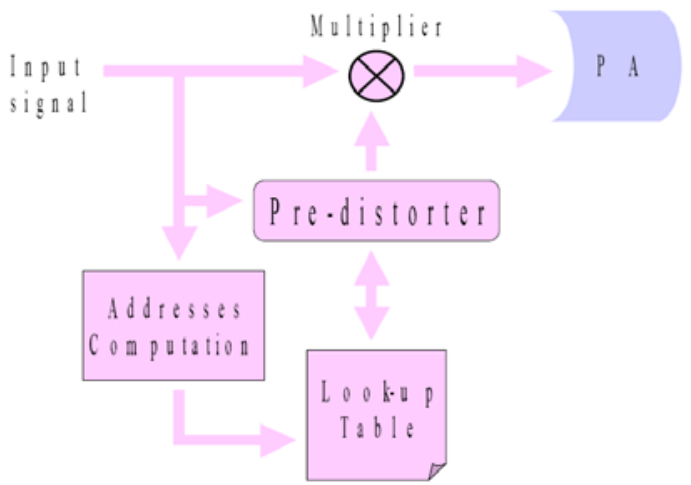

Fig. 1. The proposed system-level architecture for the lookup table based pre-distorter.

The AM-AM characteristic of the PA can be described by a mathematical function $f$, and suppose that the predistortion function of the pre-distorter is called $g$. In this work a 64QAM signal is adopted as the test signal to evaluate the envelop linearization performance. So, for one input signal called $x$, we have:

$$
f[x g(x)]=a x+b
$$

Then,

$$
x g(x)=f^{-1}(a x+b)
$$

Then,

$$
g(x)=f^{-1}(a x+b) / x
$$

where $a$ and $b$ are 2 first order polynomial coefficients computed to set up the linearized AM-AM characteristic of the PA; the computation of these 2 coefficients is detailed in what follows.

Firstly, the linear curve nearest to the AM-AM curve ( $f$ ) has to be extracted; it is represented by $a x+b$. In order to closely simulate the amplifying capability of the PA, this curve has to be as near as possible to the original one. The extraction of the linear curve from the function $f$ is done with Matlab using a first order polynomial approximation. Fig. 2. shows the AM$\mathrm{AM}$ characteristic and the linear curve extracted from it.

The Matlab function "Polyfit" returns the coefficients $a$ and $b$ of the linear approximation of the AM-AM characteristic: $a=5.9658$ and $b=-1.6148$. Please note that this linear approximation that does not cross the extremities of the AMAM characteristic but minimizes the average error that needs to be corrected. The next step is to define the correction function by computing $f^{-1}$. The AM-AM characteristic has not the same input interval than the output interval.

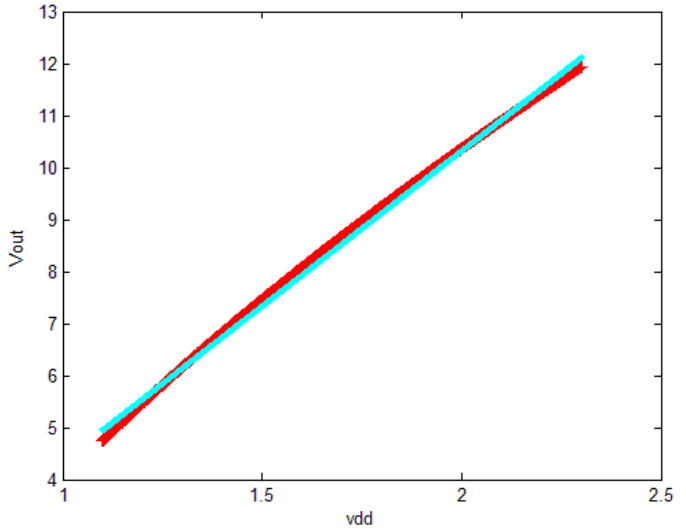

Fig. 2. AM-AM characteristic and the extracted linear curve by means of a first order polynomial approximation.

Moreover, the output interval does not entirely cover the input interval. The computation of $f^{-1}$ is possible only if $f$ is bijective. Here $f$ is bijective because it is monotonic, so $f^{-1}$ is computable. Suppose that $\mathrm{X}$ is the input interval of the AMAM characteristic, for example as in Fig. 3. for [1.1, 2.3] and that the corresponding $\mathrm{Y}$ is the output interval of the AM-AM characteristic for $[4.74,11.93]$ :

$$
\begin{aligned}
& f(x) \rightarrow Y, f^{-1}(Y)=X \\
& f(x) \in Y=[4.74,11.93] \\
& f^{-1}(y) \in X=[1.1,2.3]
\end{aligned}
$$

At this point $f^{-1}(x)$ is not completely defined, so a 3-step inversion approach is used to define it for the desired range.

The first step consists in normalizing $f$ :

$$
\begin{aligned}
& f(X)=Y \text { with } X=[1.1,11.93] \\
& f_{2}(X)=(f(X-c)-d) / e \rightarrow X
\end{aligned}
$$

where $e$ is the reduction factor of $f, d$ is the ordinate offset factor and $c$ is the abscissa offset factor, such as:

$$
\begin{aligned}
& c=\min (X) \\
& d=\min (Y) \\
& e=((\max (Y)-\min (Y)) /(\max (X)-\min (X))
\end{aligned}
$$

The second step consists in inverting the normalized function. This is possible since $f_{2}$ is a bijective and $f_{2}(X) \rightarrow X$.

In the third step, $f_{2}{ }^{-1}$ is de-normalized such as

$$
f^{-1}(X)=f_{2}^{-1}\left(e^{*} X+d\right)+c
$$

Now we compute $h=f^{-1}(a x+b)$ to modify the $\mathrm{Y}$ interval $[4.74,11.93]$ to obtain the $\mathrm{X}$ interval $[1.1,2.3]$. This modification is then canceled out by tuning $a$ and $b$ to arrive at 
the desired $h(x) . f^{-1}(Y) \rightarrow f^{-1}(X)$ is required but as $f^{-1}$ is not defined on the $X$ interval, the inverse function is offset in order to be defined on the right interval such as:

$$
f^{-1}(p \times Y+q)=f^{-1}(X)
$$

where

$p=(\max (X)-\min (X)) /(\max (Y)-\min (Y))$

$q=\operatorname{sign}(\operatorname{mean}(Y)-$ mean $(X)) *($ mean $(Y)+$ mean $(X))$

At this point $f^{-1}(a p y+a q+b)$ must be computed so that $f(H 1(x))=a 1 * x+b 1$

where $a 1$ and $b 1$ are the 2 coefficients of the desired linear function. The following system must be solved:

$$
a p y+a q+b=a 1 \times x+b 1=y
$$

One possible solution is to identify the coefficients associated with $x$ :

$$
\begin{aligned}
& a=1 / p \\
& b=-a^{*} q=1 /(p * q)
\end{aligned}
$$

Fig. 3. shows the difference between the linear curve extracted from the AM-AM characteristic (blue) and the adapted linear curve to correct the offset of the inverse function (green).

In Fig. 4. the pink curve is the AM-AM transfer function of the PA, the blue curve is the theoretical desired correction function, and the red curve is the one obtained after correction. There is a very small offset between the desired curve and the obtained one; however, this offset is insignificant as shown by the experimental results presented in section III.

\section{EXPERIMENTS}

The experiments are based on a $0.18 \mu \mathrm{m}$ polar configured CMOS Class F PA, as conceptualized in Fig. 5(A). Its transistor level simulated AM-AM and AM-PM characteristics in a polar configuration are shown in Fig. 5(B). The phase signal is applied on the input terminal while the amplitude signal is applied as Vdd (drain DC power supply terminal). The nonlinear AM-AM and AM-PM models are extracted from a transistor-level simulation and approximated with a high order polynomial.

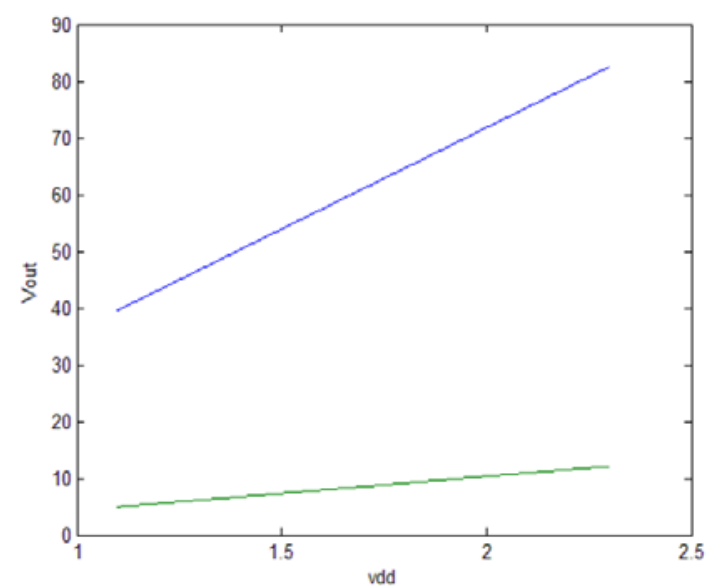

Fig. 3. Linear curve extracted from the AM-AM characteristic (blue) and the adapted linear curve to correct the offset of the inverse function (green).

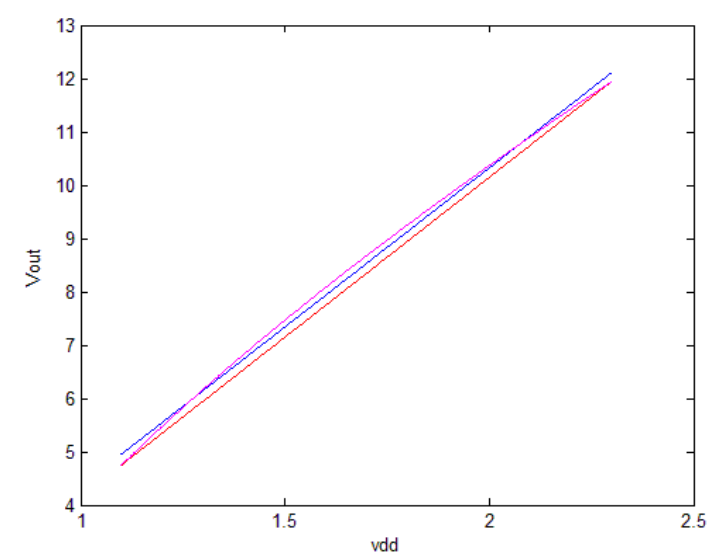

Fig. 4. AM-AM transfer function of the PA (pink), theoretical desired correction function (blue), and the obtained correction function (red).

The setup for the whole system is as depicted in Fig. 1. The signal is generated by a random data generator and modulated by a 64-QAM modulator. The 64-QAM signal (split into amplitude and phase carrier) is carried by a $2.45 \mathrm{GHz}$ carrier and fed into the Class F PA, and then down-converted to a baseband 64-QAM signal. The LUT-based predistortion function described in section II is implemented with Altera DSP-Builder for a Stratix II FPGA. The predistortion function is as shown in Fig. 6. The predistortion function is stored in a LUT memory component. The address is coded by the levels of the samples from the input signal. The predistortion value is then multiplied with the envelope of the 64-QAM signal to execute the predistortion.

Fig. 7. shows the constellations diagram for (A) the original 64-QAM signal, (B) the 64-QAM signal distorted by the PA and $(\mathrm{C})$ the 64-QAM signal linearized by the predistortion technique. Comparing Fig. 7(A) with Fig. 7(B), it is obvious that the Class F switch-mode PA distorts the signal. Fig. 7(C) shows that the presented predistortion technique improves the signal significantly. The same equalizer is applied to the signals in Fig. 7(B) and Fig. 7(C) after the amplifiers. 


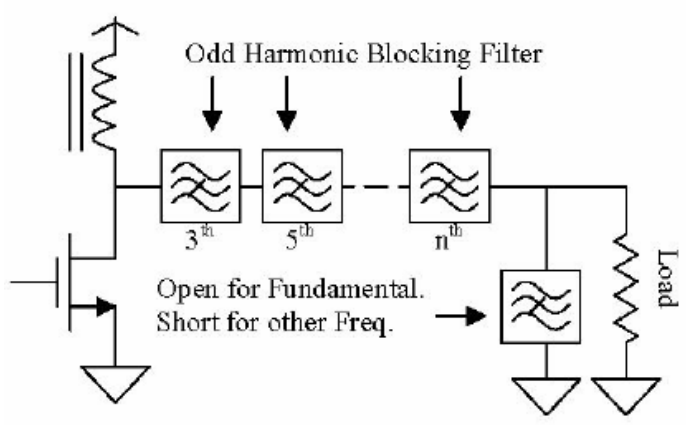

(A)

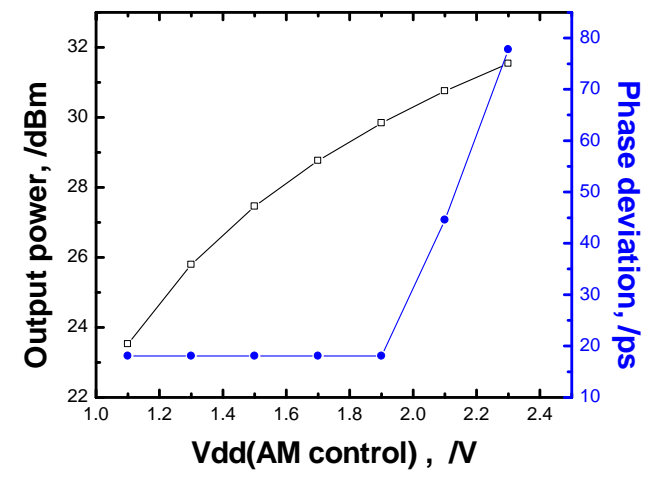

(B)

Fig. 5. A CMOS class $\mathrm{F}$ switch-mode power amplifier (A) and its transistor level simulated AM-AM and AM-PM characteristics in a polar configuration (B)

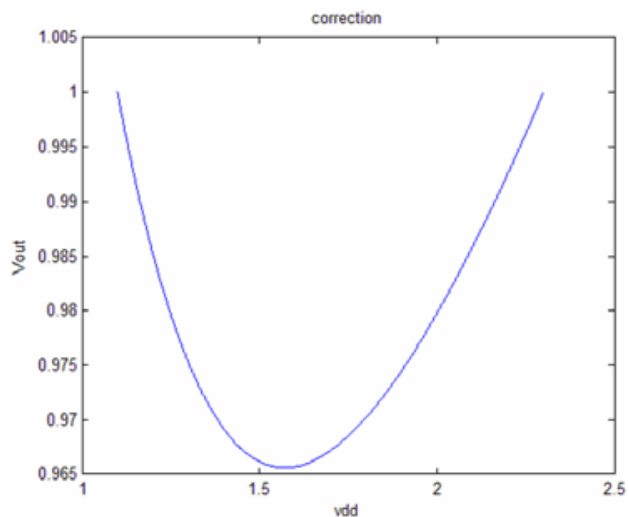

Fig. 6. The predistortion function used in practice for the experiment

Moreover, the EVM calculation (carried out on more 4000 codes samples) also shows the spectrum improvement brought by the presented linearization technique. With the presented LUT-based predistortion linearization technique, an average EVM of less than $2 \%$ is achieved.

Fig. 8 shows the spectrum improvement achieved by means of the proposed linearization. After linearization, the spectrum closing to the adjacent channel is at least $5 \mathrm{~dB}$ lower than that of the nonlinear PA.

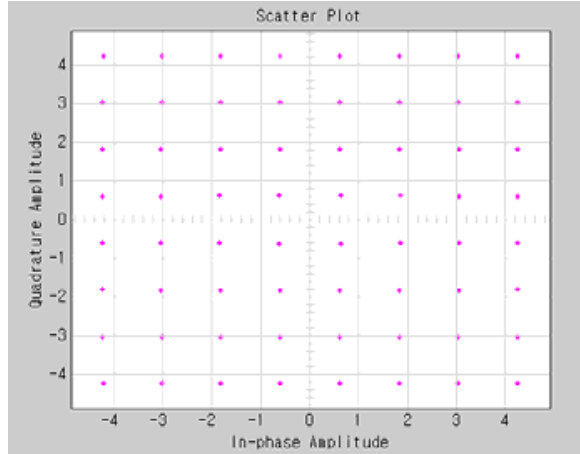

(A)

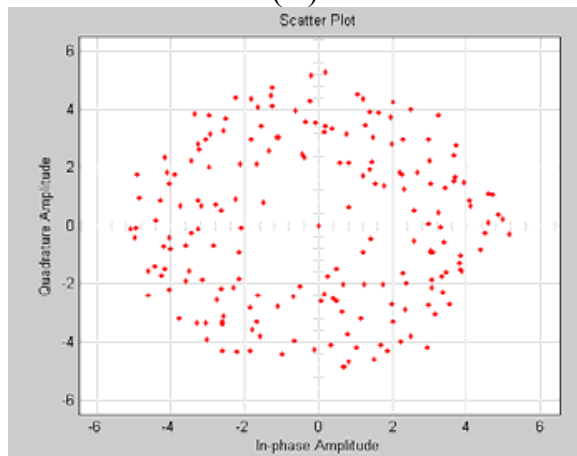

(B)

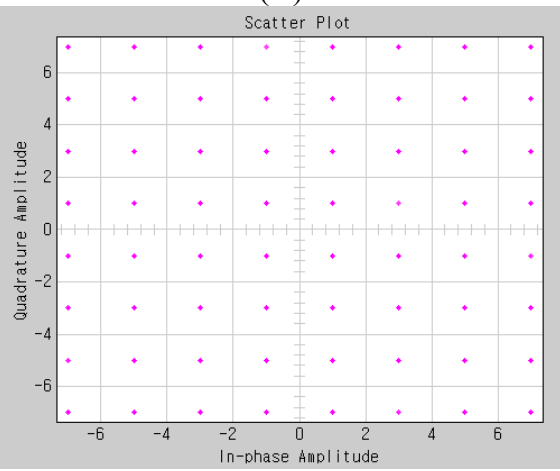

(C)

Fig. 7. Signal constellation comparison of (A) the original 64-QAM signal, (B) the distorted 64-QAM signal by the Class F power amplifier, and (C) the 64-QAM signal obtained with the presented predistortion technique.

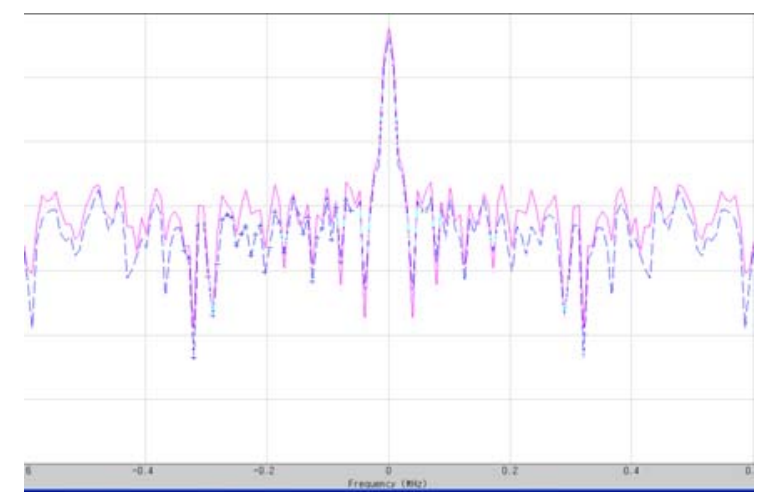

Fig. 8. The output spectrum of 64-QAM signal. The pink curve is the spectrum responded by the nonlinear PA and the blue curve is that by the linearized PA.

This is the authors' version of a paper published in the Proceedings of the 27th IEEE NORCHIP conference, 2009. 


\section{CONCLUSIONS}

A LUT-based predistortion technique targeting switchmode PA linearization has been presented. In particular we have described how the predistortion function is derived for handling the whole input interval of the nonlinearity predistortion. A $0.18 \mu \mathrm{m}$ CMOS Class $\mathrm{F}$ power amplifier is used as the study-case for testing and evaluating the proposed approach. Matlab and DSP-Builder blocks targeting a Stratix II FPGA have been used in Simulink to simulate the implementation of the predistortion function. The simulation results show that the presented LUT-based predistortion technique effectively improves the linearity of the switchmode PA. An average EVM less than $2 \%$ can be achieved based on more than 4000 codes simulation for a 64QAM signal.

\section{REFERENCES}

[1] T. S. Nielsen, "Power Amplifier Linearization Techniques for a 3rd Generation Handset”, PhD dissertation, 2005
[2] C.G.Rey, "Adaptive Polar Work Function Predistortion", IEEE Trans. Microwave Theory Tech., Vol. 47, no.6, Jun. 1999

[3] E. Westesson, L. Sundstrom, "A Complex Polynomial Predistorter Chip in CMOS for a Baseband or IF Linearization of RF power Amplifiers", in Proc. IEEE ISCAS, Vol. 1, pp.206-209, July 1999

[4] P. L. Gilabert, G. Montiro and E. Bertran, "A Methodology to Model and Predistort Short-Term Memory Nonlinearities in Power Amplifiers", Integrated Nonlinear Microwave and Millimeter-Wave Circuits, 2006 International Workshop on, Jan. 2006, pp.142 - 145

[5] N. Ceylan, J-E. Mueller and R. Weigel, "Optimization of EDGE Terminal Power Amplifiers using Memoryless Digital Predistortion", IEEE Transaction on Microwave and Technologies, Vol. 53, No. 2, February 2005, pp.515-522

[6] D. Ronnow and M. Isaksson, "Digital Predistortion of Radio Frequency Power Amplifiers using Kautz-Volterrra Model", Electronics Letters, 22nd June, 2006, Vol. 42, No.13

[7] P. L. Gilabert, A. Cesari, G. Montoro, et al., "Multi-Lookup Table FPGA Implementation of an Adaptive Digital Predistorter for Linearizing RF Power Amplifiers with Memory Effects", IEEE Transaction on Microwave and Technologies, Vol. 56, No. 2, Feb. 2008, pp.372-384

[8] A. N. D'Andrea, V. Lottici and R. Reggiannini, "RF Power Amplifier Linearization through Amplitude and Phase Predistortion", IEEE Transactions on Communications, Vol. 44, No.11, Nov. 1996, pp. 14771484 